\section{Infant psychomotor development in cases of maternal postpartum depression: Observation of a mother and baby unit}

\author{
Laure Le Treut,1,2 François Poinso,1,2 \\ Pauline Grandgeorge, 1 \\ Elisabeth Jouve, ${ }^{3}$ Michel Dugnat, ${ }^{1}$ \\ Joshua Sparrow, ${ }^{4}$ Jokthan Guivarch1,2 \\ 1Department of Child Psychiatry, Sainte \\ Marguerite Hospital APHM; ${ }^{2}$ Faculty of \\ Medicine of Marseille, Aix-Marseille \\ University; 3Department of Medical \\ Evaluation and Public Health, APHM, \\ Marseille, France; ${ }^{4}$ Brazelton \\ Touchpoints Center, Division of \\ Developmental Medicine Boston \\ Children's Hospital/Harvard Medical \\ School, Boston, MA, USA
}

\begin{abstract}
Studies of the first year of infant psychomotor development in cases of maternal postpartum depression are lacking. The mother and baby unit (MBU) is a healthcare system available to infants and their mothers during the postpartum period in a psychiatric hospital, which provides support and preserves the parent's role in the child's daily care. The aim of the paper is to describe the developmental profile of babies of mothers with severe postpartum depression treated in an MBU through the developmental quotients. Using the BrunetLézine scale, we studied six-month-old infants whose mothers were hospitalized. The study population consisted of 15 infants. The mean global developmental quotient score was 96.7. A developmental quotient lower than 80 was not observed for any of the children. We found no global psychomotor developmental delays. Despite this, the posture subscore was the area in which we observed the most difficulties. It is possible that the tonic dialogue between the mother and infant is disrupted by maternal depression.
\end{abstract}

\section{Introduction}

Perinatal psychiatry is a recent and developing field of psychiatry. It focuses on treating maternal mental disorders and promoting child development. Postpartum depression is the most frequently observed disorder during the perinatal period. ${ }^{1}$ The mother and baby unit (MBU) is a levelthree healthcare system that is available in psychiatric hospitals in France for infants and their mothers during the postpartum period. It is tasked with treating puerperal and maternal disorders, as well as relationship difficulties between mothers and infants..$^{2-4}$ The intervention used is based on the continuous presence of psychiatric and pediatric nurses for the mother and child, respectively. Then, the first principle of support preserves the parent's role in the child's daily care. The nurses adopt a reserved attitude but are available and remain present at anxious times. Nonetheless, they must accept the mother's request to take her place for either a given moment or more regularly. The limits of their neutrality depend on the following two factors: the child's protection (if the mother seems to be inadequate, violent, or insufficiently reassuring) and the mother's request for intervention. The aim of the caregivers is to provide support in the development of a maternal identity, exemplifying and serving as a role model for the attitudes, behaviors, and methods that the mother can imitate. ${ }^{2}$ Thus, they play a role in both prevention and early intervention in child development.

The indications for joint hospitalization are initial or recurring episodes of postpartum psychiatric disorders, acute or chronic maternal psychiatric pathologies, motherbaby relationship disorders, functional disorders and some infant developmental delays. $^{2-4}$

The approach to treating mothers during mother-infant hospitalizations is twofold: treatment of the psychiatric disorder and evaluation and support of the developing mother-infant interactions. ${ }^{2}$ For the infant, the institution ensures that physical and psychiatric care is safe and consistent. 5 This does not mean that the institution is replacing the mother but that it defers to the mother when she is providing the necessary care and steps in to compensate at times when maternal care is unavailable or when the pathological process hinders mother-infant interactions.

Relational challenges, even as early as the first year, have been correlated with the development of abnormal psychology in children, resulting in some cases of psychomotor retardation and difficulties in forming attachments. 6,7 Few studies have focused on evaluating the psychomotor development of babies in cases where maternal psychiatric pathology occurs during the first year. Selma Fraiberg emphasized the need to monitor the development of the baby independently of professional caregivers, since they may underestimate
Correspondence: Laure Le Treut, Department of Child Psychiatry, Sainte Marguerite Hospital, APHM, 270 Boulevard de Sainte Marguerite, 13009 Marseille, France.

Tel.: +33.491744767 - Fax: + 33491746242 .

E-mail: laure.le-treut@ap-hm.fr

Key words: Maternal postpartum depression; infant psychomotor development; revised Brunet-Lézine Scale; mother and baby unit.

Contributions: Substantial contributions to the conception or design of this work or to the acquisition, analysis, or interpretation of the data for this work: LLT, FP, PG, EJ, MD, JG. Drafting this work or critically revising it in terms of important intellectual content: LLT, FP, PG, EJ, MD, JS, JG. Final approval of the version to be published: LLT, FP, PG, EJ, MD, JS, JG. Agreement to be accountable for all aspects of this work in ensuring that questions related to the accuracy or integrity of any part of this work are appropriately investigated and resolved: LLT, FP, PG, EJ, MD, JS, JG.

Conflict of interest: the authors declare no potential conflict of interest.

Funding: none.

Received for publication: 15 June 2017.

Revision received: 27 November 2017.

Accepted for publication: 28 November 2017.

This work is licensed under a Creative Commons Attribution-NonCommercial 4.0 International License (CC BY-NC 4.0).

(C) Copyright L. Le Treut et al., 2018

Licensee PAGEPress, Italy

Mental Illness 2018; 10:7267

doi:10.4081/mi.2018.7267

delays and signs of illness in infants because of their bond with and empathy toward the troubled parent. ${ }^{8}$

In this article, we present the results of a descriptive study of a population of sixmonth-old infants whose mothers were hospitalized for severe postpartum depression in the MBU. Their development was assessed using the revised Brunet-Lézine scale, ${ }^{9}$ which is the most frequently used tool in France for the assessment of infant psychomotor development. 10 Our objective was to describe the developmental profile of babies of mothers with severe postpartum depression that were hospitalized in the MBU of the Marseille University Hospital Center using the developmental quotients. We tested our hypothesis that infants of depressed mothers would present with an atypical developmental profile, eventually resulting in a developmental delay. 


\section{Materials and Methods}

\section{Plan and population}

We performed a cross-sectional, descriptive study of the development of 6month-old infants of mothers with depression using the revised Brunet-Lézine scale.

Inclusion criteria: i) mother experienced her first severe depressive episode during the postnatal period. The diagnosis was made according to the DSM-IV's criteria by the MBU's two senior psychiatrists; ii) mother with an infant aged $6 \pm 1$ months; iii) both parents' agreement obtained for the anonymized use of their data.

Exclusion criteria: i) mothers with a different pathology (anxiety disorders, psychotic disorders, borderline disorders with no associated signs of depressive episodes); ii) infant discharged before the age of six months or admitted after the age of six months; iii) infant with a preexisting delay.

\section{Judgment criterion: the Brunet- Lézine scale}

The revised Brunet-Lézine test is one of the only development scales for young children that has been calibrated using a French sample. It is similar to the Bayley test. 11 The Brunet-Lézine scale is the test most often used in France in the field of infant developmental evaluation. Four major developmental areas are studied: posture DQ, coordination $\mathrm{DQ}$, sociability $\mathrm{DQ}$, and language $\mathrm{DQ}$.

The global developmental quotient (global DQ) is calculated from these subscores. The normal global DQ value range is from 80 to 120 , with a mean of 100 . A developmental delay is indicated if the global DQ is lower than 70. The revised Brunet-Lézine scale provides an assessment at a time $\mathrm{T}$ in the child's development.

\section{Measurement}

A psychologist who was external to the treatment team administered the BrunetLézine tests in an attempt to provide a more objective point of view on the impact of relational challenges on the baby.

\section{Statistical methods}

The statistical analysis was primarily descriptive. The qualitative variables are described by the numbers and percentages of the patients within each group. The quantitative variables (DQ and subscores) are reported as means, standard deviations and medians. The $95 \%$ confidence intervals $(95 \% \mathrm{CI})$ were calculated. Next, pairwise comparisons of the DQ scores were tested using paired t-tests, then the $\mathrm{P}$-values were corrected using the Bonferroni adjustment.

\section{Ethics Committee}

The protocol was submitted to the University of Aix-Marseilles's Ethics Committee, which granted its approval.

\section{Results}

\section{Description of the study population}

The study population consisted of 14 dyads and 15 infants (one dyad included a set of twins) successively treated in the mother and baby unit of the SaintMarguerite University Hospital Center in Marseille between 2014 and 2016.

The main diagnosis of the mothers was severe postpartum depression. Three of the 14 mothers had borderline personality disorder, and one had a depressive episode in the context of bipolar disorder. There were 15 infants comprised of ten boys and five girls. The mean age of the babies was six months and 8 days. The main characteristics of the population are presented in Table 1 .

\section{Description of the Developmental Quotients of the children}

The mean global Developmental Quotients (DQ) score was 96.7 (95\%CI: 93.4-99.9), the median was 96.8 and the range was 108 to 87 . Two children had a global DQ score under 90, but no child had a score under 80 .

Regarding the DQ subscores, the mean posture DQ score was 92.3 (median 93.7; 95\%CI: 87.2-97.4); the mean coordination DQ score was 98.2 (median 98.6; 95\%CI: 93.5-102.9); the mean language DQ score was 102.7 (median 100.5; 95\%CI: 97.8107.5); and the mean sociability DQ score was 95.5 (median 97; 95\%CI: 93.4-99.9). The main developmental scale results are displayed in Table 2 and Figure 1.

Table 1. Population characteristics.

\begin{tabular}{lcc} 
Variables & N. & $\%$ \\
Child's sex & & \\
Boy & 10 & 66.7 \\
\hline Girl & 5 & 33.3 \\
Mother's diagnosis & 10 & 71.4 \\
$\quad$ Postpartum depression & 3 & 21.4 \\
$\quad$ Postpartum depression + borderline personality disorder & 1 & 7.14 \\
$\quad$ Postpartum depression + bipolar disorder & 34 years and 8 months & \\
\hline Other parameters & 6 months and 8 days & \\
$\quad$ Mother's age, mean & & \\
Child's age, mean & & \\
\hline
\end{tabular}

\section{Comparisons of the Developmental Quotients scores}

Pairwise comparisons of the DQ scores showed differences between:

- Posture DQ and global DQ $(\mathrm{P}=0.013)$

- Posture DQ and coordination DQ $(\mathrm{P}=0.035)$

- Posture DQ and language DQ $(\mathrm{P}=0.005)$

- $\quad$ Language DQ and global DQ $(\mathrm{P}=0.016)$

- Language DQ and sociability DQ $(\mathrm{P}=0.011)$

After the Bonferroni adjustment, we found a difference between the Posture DQ and the language $\mathrm{DQ}(\mathrm{P}=0.05)$.

\section{Discussion and Conclusions}

The main result of our study was the absence of developmental delays among the infants in our population. This is a significant result, as shown by the $95 \% \mathrm{CI}$, which does not include values lower than 80 . Only two children fell in the average-low zone, with a global DQ score of 88 . These results are consistent with the observations of Piteo et al., who studied the development of 18month-old infants whose mothers suffered from postpartum depression. They found that maternal depression during the first 6 months of the postpartum period was not associated with cognitive, language or motor development in infants 18 months of age. ${ }^{12}$ We found a developmental profile characterized by higher values in the language DQ score and lower values in the posture DQ score. After adjustment, these results were significant.

Although it was not pathological, the posture category was the index wherein we observed the most difficulties in the population of children studied. Two children were actually in the borderline zone (score of 70$80)$. Four other children had results in the average-low zone (score of 80-90), 
accounting for $26.6 \%$ of the population. A total of six children, or $40 \%$, had low scores in this index.

These children of depressive mothers did not, therefore, present any impaired development in the first months of life, but some of them seemed fragile in terms of the posture category.

It is possible that the tonic dialogue described by Wallon and Ajurriaguerra is disrupted by maternal depression, both on an emotional level and through maternal psychomotor anomalies. ${ }^{13,14}$

Tonic dialogue is one of the ways to help the baby with tonic regulation, which occurs by metabolizing body tension that is accumulated in reaction to sensory stimuli and giving it meaning. For Bullinger, ${ }^{15}$ tonic dialogue meets a vital communication need for the baby. Babies are sensitive to tonic and rhythmic variations of the arms that hold them. Tonic variations in mothers affect tonic variations in babies. 16

Psychomotor anomalies are some of the most fundamental features of depression. They manifest themselves as disturbances in speech, facial expression, fine motor behavior, gross motor activity and ideation. ${ }^{17}$ In postpartum depression, psychomotor symptoms are more frequent than in non-postpartum depression.18 When tonus, rhythm, facial mimics and prosody in the mother are not congruent, the baby cannot understand the mother's emotions. ${ }^{16}$ In postpartum depression, there is a lasting impact of the mother's tonus, rhythm, and facial mimics, which disturbs tonic dialogue and could impact tonus in the baby. This effect could explain the moderate drop in the posture DQ scores.

The DQs in this study were tested early. The mothers also received psychiatric care that attenuated the severity of the depressive symptoms and, therefore, any potential effects on the child's development. Furthermore, the infants who received early treatment in the mother and child unit had the advantage of having childcare nurses

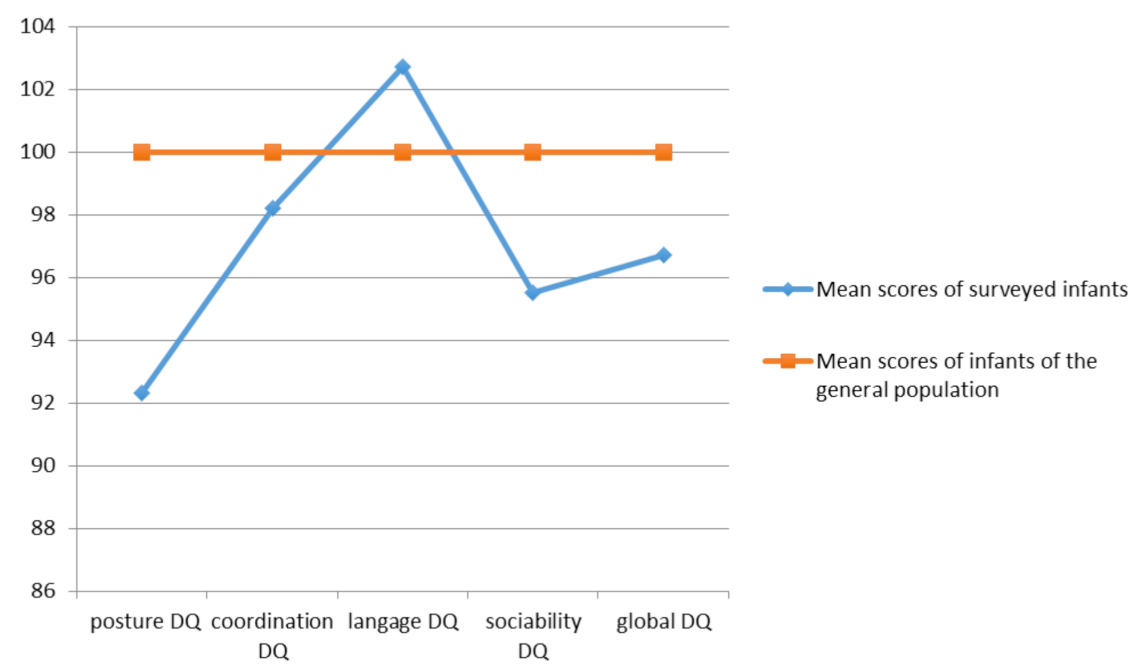

Figure 1. Mean subscores and global scores of the infants on the revised Brunet-Lézine scale. with them. It is possible that this treatment, which focused on interactions and on promoting interpersonal communication, boosted early socialization. We would, therefore, suggest that the treatment had a positive effect on infant development. Indeed, childcare nursing could allow the development of nonverbal communication and of maternal positive feelings regarding parenting. These items are the two most important factors of resilience that were identified in a prospective cohort study of children who were exposed to depressive

Table 2. Developmental Quotients scores on the revised Brunet Lézine scale.

\begin{tabular}{|c|c|c|c|c|c|c|c|}
\hline Case & Sex & Age (months) & Posture DQ & Coordination DQ & Language DQ & Sociability DQ & Global DQ \\
\hline $\mathrm{S} 1$ & M & 6 & 96 & 103 & 108 & 108 & 102 \\
\hline $\mathrm{S} 2$ & M & 6 & 98 & 98 & 114 & 98 & 100 \\
\hline S3 & M & 6 & 102 & 99 & 90 & 99 & 99 \\
\hline S4 & M & 6 & 91 & 88 & 115 & 96 & 94 \\
\hline S5 & M & 6 & 104 & 106 & 100 & 100 & 103 \\
\hline S6 & M & 6 & 95 & 100 & 100 & 84 & 96 \\
\hline S7 & M & 6 & 101 & 114 & 114 & 98 & 108 \\
\hline S8 & M & 7 & 85 & 106 & 109 & 96 & 98 \\
\hline $\mathrm{S} 9$ & $\mathrm{~F}$ & 6 & 73 & 93 & 108 & 100 & 88 \\
\hline $\mathrm{S} 10$ & M & 7 & 89 & 106 & 100 & 92 & 97 \\
\hline S11 & $\mathrm{F}$ & 6 & 99 & 103 & 99 & 99 & 102 \\
\hline S12 & $\mathrm{F}$ & 6 & 98 & 90 & 98 & 82 & 92 \\
\hline S13 & $\mathrm{F}$ & 6 & 89 & 83 & 100 & 100 & 93 \\
\hline S14 & $\mathrm{F}$ & 7 & 75 & 93 & 84 & 93 & 87 \\
\hline S15 & M & 6 & 90 & 91 & 101 & 87 & 91 \\
\hline Mean & & $6 \mathrm{~m} 8 \mathrm{~d}$ & 92.3 & 98.2 & 102.7 & 95.5 & 96.7 \\
\hline Median & & & 93.7 & 98.6 & 100.5 & 97.0 & 96.8 \\
\hline SD & & & 9.2 & 8.4 & 8.8 & 6.9 & 5.9 \\
\hline L95\%CI & & & 87.2 & 93.5 & 97.8 & 91.6 & 93.4 \\
\hline U95\%CI & & & 97.4 & 102.9 & 107.5 & 99.3 & 99.9 \\
\hline
\end{tabular}


symptoms post-natally. ${ }^{19}$. A future comparative study using a control group of depressive mothers who were treated without specific childcare would allow us to confirm this hypothesis.

The main limitation of this study was the population size due to the small size of our unit.

The DQ subscores of the different developmental areas should also be interpreted with caution because they are based on a small number of items, and the behavior of very young children is labile and remains difficult to assess, even for seasoned psychologists.

The scores taken when the babies were six months old could serve as a baseline; it might be interesting to add longitudinal timepoints to the study and to perform retests over time in order to investigate if the infant profiles are maintained, if drops in the DQ scores appear, and/or the scores correlate with maternal symptom progression.

Despite the rather reassuring appearance of our results, we would like to draw attention to the advantages of a more objective metric than the daily observation of a baby's behavior. This assessment is part of a systematic evaluation and is useful in learning a baby's strengths and weaknesses; it can also sometimes identify developmental warning signs.

This study is the first step before larger prospective and controlled studies. In another study, we would like to compare infants and mothers in a controlled setting without a mother and baby unit to study the precise impact of the unit. For these studies, it will be necessary to increase the size of our population through the participation of other mother and baby units.

\section{References}

1. Glangeaud-Freudenthal NMC, Sutter $\mathrm{AL}$, Thieulin AC, et al. Inpatient mother-and-child postpartum psychiatric care: factors associated with improvement in maternal mental health. Eur Psychiatry J Assoc Eur Psychiatr 2011; 26:215-23.

2. Poinso F, Gay MP, GlangeaudFreudenthal NMC, Rufo M. Care in a mother-baby psychiatric unit: analysis of separation at discharge. Arch Womens Ment Health 2002;5:49-58.

3. Cazas O, Glangeaud-Freudenthal NMC. The History of Mother-Baby Units (MBUs) in France and Belgium and of the French version of the Marcé checklist. Arch Women's Ment Health 2004; 7:53-8.

4. Glangeaud-Freudenthal NMC, SutterDallay AL, Thieulin AC, et al. Predictors of infant foster care in cases of maternal psychiatric disorders. Soc Psychiatry Psychiatr Epidemiol 2013;48:553-61.

5. Winnicott DW. De la pédiatrie à la psychanalyse. Paris: Payot; 2008.

6. Murray L, Arteche A, Fearon P, et al. The effects of maternal postnatal depression and child sex on academic performance at age 16 years: a developmental approach. J Child Psychol Psychiatry 2010;51:1150-9.

7. Cornish AM, McMahon CA, Ungerer JA, et al. Postnatal depression and infant cognitive and motor development in the second postnatal year: The impact of depression chronicity and infant gender. Infant Behav Dev 2005;28:407-17.

8. Fraiberg L, Fraiberg S. Clinical studies in infant mental health: the first year of life. New York: Basic books; 1980.

9. Brunet O, Lézine I, Josse D. BrunetLézine révisé: échelle de développe- ment psychomoteur de la première enfance: BLR. Editions et applications psychologiques. 1997.

10. Fily A, Pierrat V, Delporte V, Breart G, et al Factors associated with neurodevelopmental outcome at 2 years after very preterm birth: the populationbased Nord-Pas-de-Calais EPIPAGE cohort. Pediatrics 2006;117:357-66.

11. Bayley N. Bayley scales of infant development: manual. Psychological Corporation. 1993.

12. Piteo AM, Yelland LN, Makrides M. Does maternal depression predict developmental outcome in 18 month old infants? Early Hum Dev 2012;88:6515.

13. Wallon H. Les origines du caractère chez l'enfant: les préludes du sentiment de personnalité. 2015.

14. Ajuriaguerra J de. Manuel de psychiatrie de l'enfant. 2. éd. Paris: Masson; 1974. 1089 p.

15. Bullinger A. Le développement sensorimoteur de l'enfant et ses avatars: Un parcours de recherche. Ramonville Saint-Agne: Erè; 2004.

16. Bachollet M-S, Marcelli D. Le dialogue tonico-émotionnel et ses développements. Enfances Psy 2011:14-9.

17. Bennabi D, Vandel P, Papaxanthis C, et al. Psychomotor Retardation in Depression: A Systematic Review of Diagnostic, Pathophysiologic, and Therapeutic Implications. BioMed Res Int 2013;2013:158746.

18. Bernstein IH, Rush AJ, Yonkers K, et al. Symptom features of postpartum depression: are they distinct? Depress Anxiety 2008;25:20-6.

19. Savage-McGlynn E, Redshaw M, Heron J, et al. Mechanisms of Resilience in Children of Mothers Who Self-Report with Depressive Symptoms in the First Postnatal Year. PLOS One 2015;10:e0142898. 